\title{
Noncommutative Geometry, Topology and the Standard Model Vacuum
}

\author{
R. A. Dawe Martins \\ Nottingham University, University Park, \\ Nottingham, NG7 2RD, UK \\ rachel.dawe@maths.nottingham.ac.uk
}

28th August 2018

\begin{abstract}
As a ramification of a motivational discussion for previous joint work, in which equations of motion for the finite spectral action of the Standard Model were derived, we provide a new analysis of the results of the calculations therein, switching from the perspective of Spectral triple to that of Fredholm module and thus from the analogy with Riemannian geometry to the pre-metrical structure of the Noncommutative geometry. Using a suggested Noncommutative version of Morse theory together with algebraic $K$-theory to analyse the vacuum solutions, the first two summands of the algebra for the finite triple of the Standard Model arise up to Morita equivalence. We also demonstrate a new vacuum solution whose features are compatible with the physical mass matrix.
\end{abstract}




\section{Introduction}

This article is a continuation of previous work joint with Barrett [SMV] in which field equations were calculated for the full set of internal space metric fluctuations allowed by the Noncommutative geometry axioms in the spectral triple formulation of the standard model. We begin with a development of the discussion begun in the previous work, and then provide a new analysis of the results of the calculations therein from the perspective of Fredholm module instead of Spectral triple. Studying these Fredholm modules using algebraic $K$-theory and $K$-homology leads to a suggested Noncommutative version of Morse theory, - a well-known tool for studying the topology of manifolds - which is applied to the finite spectral action.

As this work ramifies from [SMV], for this article to make sense it is necessary to give an explanation of the key concepts of the previous work before the main analysis in this article can begin. Furthermore, discussion given in the previous work is brief and so one of the purposes of this paper is to explain how it highlights an open question about the Noncommutative framework. This explanation leads into a detailed introduction to the main analysis given at the beginning of the main section, entitled 'Fredholm module solutions'.

\section{Context}

More details about the tools and formalisms referred to below are provided in the background.

The context of [SMV] and this its 'second chapter', is on the spectral action principle [SAP] by Connes and Chamseddine, where the standard model is formulated with a product (whose image is called the total space) of two spectral triples:- one that represents the Euclidean space-time manifold and the other the 0-dimensional internal space of particle charges. The space-time coordinate functions remain commutative but the internal space is a noncommutative 'manifold'. The spectral action principle is an important step towards the unification of gravity with particle physics; the Einstein-Hilbert action plus Weinberg-Glashow-Salam theory all result from a calculation of the eigenvalues of the Dirac operator on the total space and since the Dirac operator encodes the metric, the spectral action principle is a purely geometrical theory.

The scope of this study involves irreducible finite real spectral triples over the complex numbers; irreducible in the sense that there is no proper invariant subspace of the Hilbert space for which the triple restricted to that space is itself a (nondegenerate) triple $\mathrm{OCI}$. For example, the standard model finite triple of three fermion families is reducible whereas the one family triple is irreducible. By finite, we mean a finite dimensional Hilbert space over a semi-simple algebra. A caveat is that calculations carried out in this and the previous article apply only in the context of these 0-dimensional geometries with Euclidean signature. This means that at present, no direct physical inference can be made. 
Currently concerned with the internal space triple by itself and not the full standard model tensor product triple, we consider a single point of space-time: we remove all terms that do not depend solely on the fluctuations of the internal space Dirac operator $D_{F}$. The finite spectral action corresponds to the Higgs potential: $\operatorname{Tr}\left(D_{F}^{4}-\right.$ $\left.2 D_{F}^{2}\right)$.

The extra Einstein's equations for internal space were calculated by Schücker et al OCI] (for one generation of elementary fermions) by minimising the Higgs potential with respect to the 'fluctuated Dirac operator' GM]. They found that the standard model Dirac operator was a solution. The construction of the fluctuated Dirac operator is carried out by beginning with a choice of initial Dirac operator (to correspond to the standard model fermion mass matrix) to satisfy the Noncommutative geometry axioms, and in analogy with the equivalence principle, fluctuating it with the standard model's internal space algebra of coordinates. In this way, the Higgs force is treated as an internal space version of gravity.

\section{Background}

\section{Standard model finite spectral triple}

A spectral triple $(\mathcal{A}, \mathcal{H}, \mathcal{D})$ provides the analogue of a Riemannian ${ }^{1}$ spin manifold to Noncommutative geometry [GM], C]. It consists of a real, involutive, not necessarily commutative algebra $\mathcal{A}$, a Hilbert space $\mathcal{H}$ : a finitely generated projective module, on which the algebra is represented, and a Dirac operator $\mathcal{D}$ that gives a notion of distance, and from which is built a differential algebra.

The geometry of any closed (even dimensional) Riemannian spin manifold can be fully described by a (real and even) spectral triple (according to the reconstruction theorem) and a noncommutative geometry is essentially the same structure but with the generalisation that the algebra of coordinates are allowed to be non-commuting [INS], $\mathrm{VBN}$.

For the standard model the internal Hilbert space is: $\mathcal{H}=\mathcal{H}_{L} \oplus \mathcal{H}_{R} \oplus \mathcal{H}_{L}^{c} \oplus \mathcal{H}_{R}^{c}$, where

$$
\begin{gathered}
\mathcal{H}_{L}=\left(\mathbb{C}^{2} \otimes \mathbb{C}^{N} \otimes \mathbb{C}^{3}\right) \oplus\left(\mathbb{C}^{2} \otimes \mathbb{C}^{N}\right) \\
\mathcal{H}_{R}=\left((\mathbb{C} \oplus \mathbb{C}) \otimes \mathbb{C}^{N} \otimes \mathbb{C}^{3}\right) \oplus\left(\mathbb{C} \otimes \mathbb{C}^{N}\right)
\end{gathered}
$$

and whose basis is labelled by the elementary fermions and their antiparticles [FCG]. The symbol $c$ is used to indicate the section represented by the antiparticles. The

\footnotetext{
${ }^{1}$ Note: Riemannian not pseudo-Riemannian; applications are to Euclidean not Lorentzian spacetimes.
} 
even triple has the $\mathbb{Z} / 2$-grading operator $\chi$, the chirality (eigenvalues +1 or -1 ). In either case of $\mathcal{H}_{L}$ and $\mathcal{H}_{R}$, the first direct summand is the quarks and the second, the leptons. $N$ stands for the number of generations. For example, the left-handed up and down quarks form an isospin doublet and their right-handed counterparts are singlets and there are three colours for quarks and none for leptons. The charges on the particles are identified by the faithful representation of the algebra on the Hilbert space.

In the definition of $\mathcal{H}$ above we see a second $\mathbb{Z} / 2$-grading that splits the Hilbert space into two orthogonal subspaces for particles and antiparticles: $\mathcal{H}^{+} \oplus \mathcal{H}^{-}$or $\mathcal{H} \oplus \mathcal{H}^{c}$ NGR. This is called $S^{o}$-reality and is not an axiom but applies to the standard model as it excludes Majorana masses. The $S^{o}$-reality grading operator $\epsilon$ satisfies: $[\mathcal{D}, \epsilon]=0,[J, \epsilon]_{+}=0, \epsilon^{*}=\epsilon, \epsilon^{2}=1$. (Compare with reality operator $J$ explanation below.)

Let $D_{F}$ denote the Dirac operator that acts on the finite dimensional internal Hilbert space; it is the internal space counterpart of the Dirac operator that acts on spacetime. $D_{F}$ is a matrix whose parameters are given by the Higgs field, CabbiboKobayashi-Maskawa family mixing matrix and the Yukawa couplings [SAP]. In other words, it provides the fermion mass matrix.

The choice made for $D_{F}$ in order that the spectral action principle reproduces the standard model is:

$$
D_{F}=\left(\begin{array}{cccc}
0 & M^{*} & 0 & 0 \\
M & 0 & 0 & 0 \\
0 & 0 & 0 & M^{T} \\
0 & 0 & \bar{M} & 0
\end{array}\right)
$$

with basis left, right, then antiparticles left and right. $M=M_{Q} \otimes 1_{3} \oplus M_{L}$, and

$$
\begin{aligned}
M_{Q} & =\left(\begin{array}{cc}
k_{u} \phi_{1} & k_{d} \phi_{2} \\
-k_{u} \bar{\phi}_{2} & k_{d} \bar{\phi}_{1}
\end{array}\right) \\
M_{L} & =\left(\begin{array}{cc}
k_{e} \phi_{1} & k_{e} \phi_{2} \\
0 & 0
\end{array}\right)
\end{aligned}
$$

(An extra row is added to $M_{L}$ here so that the matrices are square, this is not normally done and relative to other literature, the labelling $M$ is swapped with $M^{*}$.)

with

$$
k_{u}=\left(\begin{array}{ccc}
m_{u} & 0 & 0 \\
0 & m_{c} & 0 \\
0 & 0 & m_{t}
\end{array}\right) \quad k_{d}=V_{C K M}\left(\begin{array}{ccc}
m_{d} & 0 & 0 \\
0 & m_{s} & 0 \\
0 & 0 & m_{b}
\end{array}\right)
$$




$$
k_{e}=\left(\begin{array}{ccc}
m_{e} & 0 & 0 \\
0 & m_{\mu} & 0 \\
0 & 0 & m_{\tau}
\end{array}\right)
$$

$T$ denotes transposition, $*$ denotes hermitian conjugation, bar denotes complex conjugation, $m_{x}$ are the Yukawa couplings of the elementary fermions, $V_{C K M}$ is the Cabibbo-Kobayashi-Maskawa generation mixing matrix. $\left(\phi_{1}, \phi_{2}\right)^{T}$ is the (Higgs) scalar doublet.

The finite spectral action corresponds to the Higgs potential: $\operatorname{Tr}\left(D_{F}^{4}-2 D_{F}^{2}\right)$. If the $\operatorname{Tr}(I)$ term is included [SAP], which obviously does not affect the equations of motion, then the action can be written $\operatorname{Tr}\left(M M^{*}-I\right)^{2}$.

The spectral triple algebra $\mathcal{A}$ is a subalgebra of the bounded operators on the Hilbert space, it is a $*$-algebra not necessarily a $C^{*}$-algebra but its norm-closure in the Hilbert space is a $C^{*}$-algebra. The standard model tensor product algebra is 'almost commutative':

$$
\mathcal{A}=C^{\infty}(M) \otimes\left(\mathbb{H} \oplus \mathbb{C} \oplus M_{3}(\mathbb{C})\right)
$$

where the first factor is the (commutative) algebra of function on (Euclidean) spacetime and the second factor is internal space algebra of particle charges.

The (faithful) representation $\rho$ of the finite space algebra has been worked out by Connes to correspond to the particle charges see [SAP]. The first and second summand acts on the particles while the third summand acts on the antiparticles. The basis is given by the Hilbert space above.

$$
\rho:=\left(\begin{array}{cccc}
\rho_{L} & 0 & 0 & 0 \\
0 & \rho_{R} & 0 & 0 \\
0 & 0 & \rho^{c} & 0 \\
0 & 0 & 0 & \rho^{c}
\end{array}\right)
$$

\section{Real structure and Poincaré duality}

Instead of splitting $\mathbb{C}$ into two copies of $\mathbb{R}$ as its name suggests, $J$ forms two subspaces of the Hilbert space (not orthogonal as in $S^{\circ}$-reality), which are interpreted as fermions and antifermions, in which case $J$ is given by the composition of the charge conjugation operator with complex conjugation. The mathematical purpose of the $J$ operator entering the axioms is to provide Connes' notion of a 'noncommutative manifold'. That is, by turning the Hilbert space into a bimodule, the 'real' structure ([NGR] allows the generalisation of Poincaré duality to the spectral triple; $a \in \mathcal{A}$ and the opposite algebra, $b^{o} \in \mathcal{A}^{o}$ (or $\mathcal{A}^{o p}$ ) where $a$ acts on the left of $\mathcal{H}$ and $b^{o}$ acts on the right. 


$$
\left[a, b^{o}\right]=0, \quad b^{o}=J b^{*} J^{-1} \quad \forall a, b \in \mathcal{A}
$$

The opposite algebra provides the 'dual' to the algebra. The pairing of the $K$-theories of these two algebras provides a noncommutative geometric version of Poincaré duality. This structure is also important in the notion of first order differential operator in noncommutative geometry. The tangent space over any manifold is real, and the reality structure gives rise to the real- $K$-theory of the enveloping algebra $\mathcal{A} \otimes \mathcal{A}^{o}$.

The action of $J$ on $\mathcal{H}$ as given by the composition of charge conjugation and complex conjugation:

$$
J\left(\begin{array}{l}
\psi_{1} \\
\bar{\psi}_{2}
\end{array}\right)=\left(\begin{array}{l}
\psi_{2} \\
\bar{\psi}_{1}
\end{array}\right) \quad\left(\psi_{1}, \bar{\psi}_{2}\right) \in \mathcal{H} \oplus \mathcal{H}^{c}
$$

where the bar indicates complex conjugation.

\section{$K$-theory}

$K$-theory is a generalised cohomology theory. Topological $K$-theory is the topological invariant that classifies the vector bundles over a given field, over a compact topological space $X$ up to stable equivalence. It is an abelian group $K^{0}(X)$ generated by the isomorphism classes of vector bundles over a given field. Addition is given by $[E]+[F]=[E \oplus F]$ where $[E]$ and $[F]$ are isomorphism classes of vector bundles $E$ and $F$. Every element of the group is a difference: $[E]-[F]$. The Serre-Swan theorem provides the identification of topological with algebraic $K$-theory. That is, $K^{0}(X)$ is isomorphic to the algebraic $K$-theory group $K_{0}\left(C^{0}(X)\right)$. The group $K_{0}(A)$ for a $C^{*}$-algebra $A$ is generated by the projections (self-adjoint idempotents) in $A$. These projections form an abelian semigroup rather than a group, but the Grothendieck construction turns them into an abelian group using an equivalence relation, which is very much analogous to the process of constructing the integers from the natural numbers $[\mathrm{L}, \mathrm{W}]$.

Some rules for $K$-theory include:

$K_{0}\left(M_{n}(A)\right)=K_{0}(A)$ (Morita equivalent algebras have the same $K$-theory), $K_{0}(A \oplus B)=K_{0}(A) \oplus K_{0}(B)$ where $A$ and $B$ are $C^{*}$-algebras.

\section{Fredholm modules and K-homology}

The Fredholm module is the 'pre-metric' structure that is used to define the noncommutative calculus $\mathrm{C}$. A spectral triple can be thought of as an unbounded (unless the Hilbert space is finite dimensional) Fredholm module with Dirac operator providing a notion of distance. 
Definition: Fredholm operator. A Fredholm operator is a bounded operator on a Hilbert space whose kernel and cokernel are finite dimensional and is invertible modulo compact operators.

\section{Definition: Fredholm module. C}

Let $A$ be an involutive algebra (over $\mathbb{C}$ ). Then a Fredholm module $(H, F)$ over $A$ is given by: 1) an involutive representative $\rho$ of $A$ in a Hilbert space $H$. 2) a Fredholm operator $F=F^{*}, F^{2}=I$ on $H$ such that $[F, \rho(a)]$ is a compact operator for any $a \in$ A. $^{2}$

If there is a $\mathbb{Z} / 2$ grading $\chi$, such that $\chi=\chi^{*}, \chi^{2}=1$ of the Hilbert space such that a) $[\chi, \rho]=0 \quad \forall a \in A$ and b) the anticommutator $[\chi, F]_{+}=0$ then the Fredholm is even.

There is a natural assignment of a Fredholm module to a spectral triple. To be precise, an observation given in $[\mathrm{C}]$ is that there is a canonical assignment of a Fredholm module to a spectral triple given by $F=\operatorname{sign}(\mathcal{D})$ (that is, $\mathcal{D}=F|\mathcal{D}|$ ) outside the kernel of $\mathcal{D}$. (On the finite dimensional kernel of $\mathcal{D}$, one takes an arbitrary isometry BNG].)

Kasparov's $K$-homology is the Poincaré dual theory to $K$-theory:- the $K$-homology (abelian) group of a Fredholm module is given by the homotopy classes of its Fredholm operator $F$. Let $F$ be an elliptic operator on a compact space $X$ (all such are Fredholm), then there is an isomorphism, index: $[X, F] \rightarrow K_{0}(X)$ where [,] denotes homotopy equivalence classes. Due to Connes' construction of Poincaré duality for noncommutative spaces, in which the dual to $\mathcal{A}$ is its opposite algebra $\mathcal{A}^{\text {op }}$, one can write that $[F] \in \rho\left(A^{o p}\right)$ because the abelian group $K^{0}\left(A^{o p}\right)$ is generated by the minimal rank projections of the opposite algebra $A^{o p}$. $\mathrm{AKH}$.

\section{Index and intersection form}

We recall that every finite dimensional real involutive algebra on a finite dimensional Hilbert space [CFS] over the complex numbers is isomorphic to the direct summand $M_{n_{1}}(\mathbb{C}) \oplus \ldots \oplus M_{n_{k}}(\mathbb{C})$ for some integers $n_{1}$ to $n_{k}$. Consider the Hilbert space to be made up of separate 'chunks' where each is acted upon by a different algebra summand $[\mathrm{BNG}$ :

$$
H_{i j}=P_{i} \mathcal{H} P_{j}, \quad \mathcal{H}=\bigoplus_{i, j} H_{i j}
$$

where the $P_{i}$ or $p_{i}$ are projections in $\mathcal{A}$ and the $P_{j}$ or $J p_{j} J^{-1}$ are projections in $\mathcal{A}^{o}$. The action on $H_{i j}$ from the left is $a_{i} \otimes 1 \otimes 1$ and the action from the right is $1 \otimes 1 \otimes a_{j}^{T}$.

\footnotetext{
${ }^{2}$ After some trivial changes that we do not need to go into details of here, Connes ensures that the Fredholm module makes sense in finite dimensions [C].
} 
Let $r_{i j}$ be the number of particles represented by $H_{i j}$ and $\chi$. The intersection form is:

$$
\mu_{i j}=r_{i j} \chi
$$

which has non-zero determinant.

The matrix $\mu_{i j}=r_{i j} \chi$ is the same thing as the tensor product pairing of the $K$-theory groups of the algebra $\mathcal{A}$ with its opposite algebra:

$$
\mu_{i j}=\operatorname{Tr}\left(\chi\left(\rho\left(p_{i}\right) J \rho\left(p_{j}\right) J^{-1}\right)\right)
$$

and we also see that:

$$
\mu_{i j}=r_{i j} \chi=\operatorname{dim} H_{i j}^{R}-\operatorname{dim} H_{i j}^{L}
$$

and then by summing over all the $H_{i j}$ one arrives at right-hand side of the Fredholm index formula:

$$
\operatorname{Index}\left(P \mathcal{D}^{+} P\right)=\operatorname{dim} \mathcal{H}_{R}-\operatorname{dim} \mathcal{H}_{L}
$$

where $\mathcal{D}^{+}=M^{*}$ in our conventions for the finite triple.

\section{The axioms}

Axioms 1, 3 and 5 are identical with those of commutative geometry. See GM for a full statement and explanation of the axioms.

1. $n>0 d s=\mathcal{D}^{-1}$ is an infinitesimal of order $\frac{1}{n}$ where $n$ is the dimension of the space.

2. $\left[[\mathcal{D}, a], b^{0}\right]=0 \forall a, b \in \mathcal{A}$. By axiom 7 we also have: $\left[\left[\mathcal{D}, b^{0}\right], a\right]=0 b^{o} \in \mathcal{A}^{o}$ opposite algebra.

3. (Smoothness) This is the algebraic formulation of smoothness of coordinates.

4. (Orientability) There is a Hochschild cycle $c$. For $n$ even, its representation on $\mathcal{H}$ is $\pi(c)=a^{0}\left[\mathcal{D}, a^{1}\right] \ldots\left[\mathcal{D}, a^{n}\right]$. This defines the construction of the analogue of the differential form that does not require a previous knowledge of the tangent bundle. If $n$ is odd, require $\pi(c)=1$. If $\mathrm{n}$ is even, $\pi(c)=\chi$ satisfies: $\chi=\chi^{*}$, $\chi^{2}=1, \chi D=-D \chi$ 
5. (Finiteness and absolute continuity) The Hilbert space is a finite, projective $\mathcal{A}$-module possessing a hermitian structure.

6. There is the Poincaré duality isomorphism $K_{*}(\mathcal{A}) \rightarrow K^{*}(\mathcal{A})$ where the intersection form is nondegenerate.

7. (Reality) There is an antilinear isometry $J: \mathcal{H} \rightarrow \mathcal{H}$ with $b^{0}=J b^{*} J^{-1}$ and $\left[a, b^{0}\right]=0$. The operator $J$ must satisfy a set of further conditions, which for 0-dimensions are the following. $J^{2}=I, J D=D J, J \chi=\chi J$.

\section{Gravity and internal space}

In order to motivate the main analysis of this article we give an outline of the previous work in [SMV] and discuss some of its implications. We also prove a new result.

The article [SMV] highlights the following issue. Einstein's equations involve all fluctuations of the space-time metric, and so if we believe that noncommutataive spectral triples are analogous to Riemannian spin manifolds, then we should vary the finite action with respect to the most general internal space Dirac operator allowed by the Noncommutative geometry axioms. In other words, since Riemannian geometry gives rise to the study of gravity, we should continue to treat the Higgs force as an internal space version of gravity by calculating the extra Einstein's equations for the entire set of metric fluctuations. A feature of this approach is that the element of choice is removed; the hypothesis was that the standard model fermion mass matrix would arise as a solution of these equations of motion, just as Newton's laws of motion are selected through an action minimisation principle, and thus the existence of classical mechanics can be explained mathematically. The physical mass matrix did not turn out to be a solution, in fact the additional fluctuations over-constrained the vacuum so that the solutions were completely degenerate. However, given the logic of this idea (of Barrett's), despite giving an unphysical result, it deserves further attention.

In response to the result, we may consider:

(a) abandoning the paradigm that noncommutative spectral triples be completely analogous to Riemannian geometry, and taking the Yukawa couplings to be derived from 'finely-tuned' constants,

(b) proposing that the extra fluctuations are physical, in which case additional scalar field terms in the action are needed (in order that the mass matrix vacuum be nondegenerate $)^{3}$, together with an additional internal space discrete version of gravity. Such a new interaction might arise from a background source term or a twisting of the Dirac operator,

\footnotetext{
${ }^{3}$ One replaces the action $\operatorname{Tr}\left(M M^{*}-I\right)^{2}$ with $\operatorname{Tr}\left(M M^{*}+X X^{*}-I\right)^{2}$ for some matrix $X$,
} 
(c) proposing an eighth axiom for Noncommutative geometry to act as a further geometric constraint on the Dirac operator, which might involve a definition of curvature of internal space.

Even by leaving out the $S^{\circ}$-reality condition to increase the number of degrees of freedom in the Dirac operator (connecting anti-particles with particles) and imposing the first order condition as a geometric constraint upon it, did not lead to a vacuum $M$ for the physical mass matrix because the extra fields all had zero vacuum expectation values as shown in SMV]. There is another side of the coin revealed by modifying this calculation such that those new fields are treated as constant numbers, which means that the Yukawa couplings determine from constants, or are constants as in the standard model: while the fermion mass ratios are not determined of course, the vacuum solution is a non-degenerate matrix and the product $M M^{*}$ is diagonal whereas $M^{*} M$ is not diagonal. These features are compatible with the physical mass matrix and with consideration (a) above. After defining some notation we give the proof.

We label the additional degrees of freedom in the standard model Dirac operator on internal space when $S^{o}$-reality condition is omitted: $g, u, x, h, v, y$. In [NGL] these 'leptoquarks' are variable fields and effect spontaneous breaking of colour symmetry, whereas we are treating them as constants, so here colour symmetry remains intact. There are also two more fields $j, l$ that arise exist when $\nu_{R}$ is included [SMV], which was done to allow the neutrino a mass.

We use the notation: $M=M_{Q} \otimes 1_{3} \oplus M_{L}$ with

$$
M_{Q}=\left(\begin{array}{cc}
a & b \\
c & d
\end{array}\right), \quad M_{L}=\left(\begin{array}{cc}
q & r \\
s & t
\end{array}\right)
$$

If $M$ is a diagonal matrix then $a, d, q, t$ are interpreted as Dirac masses for the up, down, electron and neutrino respectively.

The field equations ([SMV] ) for the Dirac operator with leptoquarks held constant are given in the Appendix. With $M_{Q}$ invertible ${ }^{4}$ and with all of the additional fields held constant and $r=s=0$ by gauge freedom the equations [SMV] reduce to:

$$
\begin{array}{r}
3|a|^{2}+3|b|^{2}+|g|^{2}+|u|^{2}+|x|^{2}+|h|^{2}+|v|^{2}+|y|^{2}-3=0 \\
|d|^{2}+|c|^{2}-1=0 \\
a \bar{c}+\bar{b} d=0 \\
|q|^{2}+|g|^{2}+|u|^{2}+|x|^{2}+|j|^{2}-1=0 \\
|t|^{2}+|h|^{2}+|v|^{2}+|y|^{2}+3|l|^{2}+|j|^{2}-1=0 \\
\bar{g} h+\bar{u} v+\bar{x} y=0, \quad l j=0 .
\end{array}
$$

\footnotetext{
${ }^{4}$ substituting $a d-b c=0$ into the equations for $M_{Q} 37$ to 40 gives $g=u=x=h=v=y=0$
} 
As claimed above, these equations give $M$ a non-degenerate set of eigenvalues with $M M^{*}$ diagonal and $M^{*} M$ not diagonal. In the case of the standard model where $\nu_{R}=0$, the equations are the above minus the equation involving $t$, and with $j=$ $l=0$. (In the previous work $g=u=x=h=v=y$ were allowed to vary, thus there were more equations and the solution was a fully degenerate mass matrix.)

Since $|a|^{2}+|b|^{2}$ is identified with $m_{u}\left(\left|\phi_{1}\right|^{2}+\left|\phi_{2}\right|^{2}\right)$, when $g, u, x, h, v, y$ are constants means that the Yukawa couplings are determined from numbers that are constant. The conclusion is that we have found equations of motion for which there exists a solution that is not demonstrably incompatible with experiment by means of an action principle in which an element of human choice is removed. The result ironically provides a mathematical reason for the Yukawa couplings to require fine-tuning, however options (b) and (c) are open and there is the caveat that these results apply only to the 0-dimensional, Euclidean case. Also, there are the extra particleantiparticle mixing action terms [NGL].

There is also a set of equations where $j$ and $l$ are allowed to vary while the 'leptoquarks' remain constant. These are the first three equations above (91) together with:

$$
\begin{aligned}
j & =0 \\
|q|^{2}+|g|^{2}+|u|^{2}+|x|^{2}-1 & =0 \\
|t|=|l| & =\frac{1}{2} \\
\bar{g} h+\bar{u} v+\bar{x} y & =0
\end{aligned}
$$

The conclusion is the same as above.

\section{Fredholm module solutions}

The final discussion point we would like to make with regard to [SMV] will motivate the calculations that follow. Below we make the observation that the vacuum solutions to the field equations for the full set of metric fluctuations do not pertain to the spectral triple, but rather to the pre-metric structure of the spectral triple, namely the Fredholm module. Rather than the standard model Dirac operator being a solution, its sign is a solution. We refer to the observation of Connes that any spectral triple has a Fredholm module associated to it where the Fredholm operator $F$ of the Fredholm module is identified with the sign of the Dirac operator (outside its kernel) of the spectral triple [C]. In switching focus from spectral triple to Fredholm module, one zooms out from the geometry to the topology. Hence, instead of hypothesising that the equations of motion single out the correct metric, in this section we ask if the equations of motion can give solutions which relate to topological invariants, that is, $K$-theory and $K$-homology. 
Since the $K$-homology of a Fredholm module is isomorphic to the $K$-theory of the algebra it is over, we should observe a relationship between a given algebra and the vacuum solution. Connes' realisation of Poincaré duality in Noncommutative geometry is to define the Poincaré dual to be the opposite algebra. This means that the homotopy classes of the projections in the dual algebra are identified with the $K$-homology, which we recall is given by the homotopy classes of the Fredholm module $[F]$. For a given algebra, one may identify a corresponding Fredholm module solution, and below we demonstrate this for the standard model and for one other algebra. This is only an observation, but to use this framework to obtain topological data from the vacua, we need to make the procedure unique, so that there is a oneto-one relationship between algebra and vacuum solution. To this end, tools from Morse theory are borrowed from commutative geometry and a way to generalise them for this Noncommutative work is suggested.

\section{1 $\quad S^{o}$-real standard model finite triple vacuum}

First we recall that the most general internal space Dirac operator given the appropriate constraints of self-adjointness, same dynamics for particles and antiparticles, orientability, $S^{o}$-reality and first order condition:-

$$
\begin{array}{r}
\mathcal{D}=\mathcal{D}^{*}, \quad[\mathcal{D}, J]=0, \quad[\mathcal{D}, \chi]_{+}=0, \\
{[\mathcal{D}, \epsilon]=0, \quad\left[[\mathcal{D}, a], b^{o}\right]=0, \quad\left[\left[\mathcal{D}, b^{o}\right], a\right]=0}
\end{array}
$$

(where $[,]_{+}$denotes the anticommutator)

is:

$$
D_{F}=\left(\begin{array}{cccc}
0 & M^{*} & 0 & 0 \\
M & 0 & 0 & 0 \\
0 & 0 & 0 & M^{T} \\
0 & 0 & \bar{M} & 0
\end{array}\right)
$$

where $M=M_{Q} \otimes 1_{3} \oplus M_{L}$, and we have allowed for the inclusion of $\nu_{R}$. To exclude $\nu_{R}$ as in the standard model, we simply delete the final column from $M^{*}$ (or row from $M)$.

From the first order condition,

$$
\left(M \rho^{c}-\rho^{c} M\right) \rho_{L}^{T^{\prime}}-\rho_{R}^{T^{\prime}}\left(M \rho^{c}-\rho^{c} M\right)=0
$$

when $\rho_{L}^{T^{\prime}}=0$ and $\rho_{R}^{T^{\prime}}=1$ we find that $\left[M, \rho^{c}\right]=0$ (the Higgs has no colour charge) and with the standard model representation this splits $M$ up into the direct sum of 
quark and lepton masses. Further than this, the mass matrix $M$ is not constrained by the first order condition. This means that the action does not know how the algebra is represented, and hence it is missing some geometrical information about the manifold. When $S^{o}$-reality is omitted some of this information becomes available to the action and hence (when the extra fields are kept constant) the non-degenerate mass matrix solution of the previous section arises.

As in the previous work, we drop the $S^{o}$-reality condition, and allow degrees of freedom in $D_{F}$ to vary but we leave the first order condition until after the equations of motion have been derived; since we are to aim to develop a method to identify the algebra given a vacuum solution, we had better omit any axioms that involve the algebra. However, we retain the condition that $\left[M, \rho^{c}\right]=0$.

The result is:

$$
D_{F}=\left(\begin{array}{cc}
Y & Z \\
\bar{Z} & \bar{Y}
\end{array}\right)=\left(\begin{array}{cccc}
0 & M^{*} & 0 & G \\
M & 0 & G^{T} & 0 \\
0 & \bar{G} & 0 & M^{T} \\
G^{*} & 0 & \bar{M} & 0
\end{array}\right)
$$

(where $G$ having the same dimensionality as $M$, was not a general matrix in SMV] but was constrained by the first order condition.) Alternate blocks are zero due to the condition $\left[D_{F}, \chi\right]_{+}=0$. Here we are not using the first order condition, so $G$ and $M$ are both general matrices with complex coefficients and having dimensionality depending on the number of fermions considered.

To calculate the equations of motion we vary the finite spectral action with respect the degrees of freedom in $D_{F}$ as given above, first for the $S^{\circ}$-real case and then for the non- $S^{o}$-real triple. The result of the former is the same as that given in SMV] but we make a new interpretation of it.

\subsection{1 $S^{o}$-real triple}

The action is:

$$
S=\operatorname{Tr}\left[\left(D_{F}\right)^{4}-2 D_{F}^{2}\right]
$$

or, with the $\operatorname{Tr}(I)$ term included:

$$
S=\operatorname{Tr}\left(M M^{*}-I\right)^{2}
$$

(where $I$ denotes the unit matrix) 
Minimising the 19 with respect to $M$ gave the very definition of partial isometry:

$$
M^{*}\left(M M^{*}-I\right)=0
$$

and hermitian conjugate.

This result means that the mass matrix that minimises the action gives each fermion an identical mass. The new interpretation we give is that the standard model finite triple's Dirac operator is a solution only up to its sign, and hence only up to the

conformal structure of the spectral triple, where $\operatorname{sign} D_{F}=\frac{D_{F}}{\left|D_{F}\right|}$. Specifically, the vacuum solution $M_{v a c}^{*}$ is the partial isometry in the polar decomposition of $D^{+}$. This operator $\operatorname{sign} D_{F}$ is the Fredholm operator for the Fredholm module associated to the standard model finite spectral triple outside the kernel of the Dirac operator ([C]).

\subsubsection{Non- $S^{o}$-real triple}

The action is:

$$
S=\operatorname{Tr}\left[\left(D_{F}\right)^{4}-2 D_{F}^{2}\right]
$$

with $D_{F}$ given by 17

Simplifying the action by using cyclicity of trace and the fact that $\operatorname{Tr}(X)=\operatorname{Tr}\left(X^{T}\right)$ :

$S=\operatorname{Tr}\left(-2\left(G^{*} G+M M^{*}\right)+\left(M M^{*}\right)^{2}+\left(G^{*} G\right)^{2}+2\left(M G G^{*} M^{*}+M M^{*} G^{T} \bar{G}+M G \bar{M} \bar{G}\right)\right)$

or:

$$
S=\operatorname{Tr}\left(-2\left(G G^{*}+M^{*} M\right)+\left(M^{*} M\right)^{2}+\left(G G^{*}\right)^{2}+2\left(G G^{*} M^{*} M+G \bar{M} M^{T} G^{*}+G \bar{M} \bar{G} M\right)\right)
$$

We vary the first of the above (22) with respect to $M$ and the result is:

$$
M^{*}\left(M M^{*}+G^{T} \bar{G}-I\right)+G \bar{M} \bar{G}+G G^{*} M^{*}=0
$$

and the second with respect to $G$ and the result is:

$$
G^{*}\left(G G^{*}+M^{*} M-I\right)+\bar{M} \bar{G} M+\bar{M} M^{T} G^{*}=0
$$


The field equations for $G^{T}, M^{T}, G^{*}, M^{*}, \bar{G}$ and $\bar{M}$ are just the the transpose, hermitian conjugate or complex conjugate respectively of the above equations for $M$ and $G$.

Although there are zeroes in $D_{F}$ due to orientability, the equation below is the same thing as that above, due to there being no linear terms in $M$ or $G$ :

$$
D_{F}^{3}=D_{F}
$$

which is of course the result of differentiating [18 with respect to $D_{F}$. In other words, simply by substituting for $D_{F}$ with 17 into $D_{F}^{3}=D_{F}$, precisely the equations of motion obtained above together with all their conjugate counterparts, appear.

The conclusion in this non- $S^{o}$-real case is the same as that in the $S^{o}$-real one, namely that the solutions are partial isometries. Here is the proof:-

First we check if the equations of motion do have any Fredholm module solutions. To do this, we must look for solutions such that $D_{F, v a c}^{2}=I$ :

$$
D_{F, v a c}^{2}=\left(\begin{array}{cccc}
M^{*} M+G G^{*} & 0 & M^{*} G^{T}+G \bar{M} & 0 \\
0 & M M^{*}+G^{T} \bar{G} & 0 & M G+G^{T} M^{T} \\
\bar{G} M+M^{T} G^{*} & 0 & \bar{G} G^{T}+M^{T} \bar{M} & 0 \\
0 & G^{*} M^{*}+\bar{M} \bar{G} & 0 & G^{*} G+\bar{M} M^{T}
\end{array}\right)
$$

The equations 24] and 25] (and conjugates) are equivalent to the equation $D_{F}^{3}=D_{F}$, and therefore we can state that the eigenvalues of the vacuum solution for $D_{F}$ are all in the set $\{-1,0,1\}$. Then we see that $D_{F, v a c}^{2}$ has eigenvalues all 1 or 0 , which means that assuming it is diagonalisable, $D_{F, v a c}^{2}=U p U^{*}$ for some unitary matrix $U$ and where $p$ is a projection, that is, $p$ satisfies $p=p^{2}$ and $p=p^{*}$. Clearly, $U p U^{*}$ is a projection, in other words $D_{F, v a c}^{2}$ is a projection, and since the Dirac operator is self-adoint, we may conclude that all the vacuum solutions are that $D_{F, v a c}$ is a partial isometry: $\left(D_{F, v a c}^{*} D_{F, v a c}\right)\left(D_{F, v a c}^{*} D_{F, v a c}\right)=D_{F, v a c}^{*} D_{F, v a c}$. A simpler way to see this is to multiply on both sides of the equation $D_{F, v a c}^{3}=D_{F, v a c}$ by $D_{F, v a c}$ while recalling that the Dirac operator is self-adjoint. Notice also that for the eigenvalues of $D_{F, v a c}$ that are 1, equation [26] shows that the sum of the two types of masses for each particle add up to 1 and so even if $G$ can lift the degeneracy of the Dirac mass matrix, the total mass ends up being the same. To summarise, the Fredholm module interpretation (2.1.1) is again valid in this, the non- $S^{\circ}$-real case.

\subsection{Orthogonal complements}

Although the orthogonal complement relationship between $M$ and $G$ can already be seen from the result of the last part, below we demonstrate it explicitly and analyse the equations 25] and 24. This datum will be used in the next part. 
Since a partial isometry is just a projection multiplied by a unitary matrix, we let $M$ be a diagonal projection of dimensionality $n$. (For a partial isometry $v, v \mathcal{H}=$ $\left.v v^{*} \mathcal{H}\right)$. We do not assume that $G$ is diagonal. In any calculation we assume that the dimensionalities of the matrices $M$ and $G$ are the same.

From 24 and 25 it is immediately clear that when $M=0, G$ is a partial isometry and vice versa.

With $M$ a diagonal projection inserted, the equation of motion for $M$ simplifies to:

$$
M^{*} G^{T} \bar{G}+G \bar{M} \bar{G}+G G^{*} M^{*}=0
$$

If $M=I$ it simplifies further to:

$$
G^{T} \bar{G}+G \bar{G}+G G^{*}=0
$$

and whereas if $M$ is a diagonal projection of dimensionality $n$ and rank $m$ then the bottom $n-m$ rows of 27 disappear.

We show below that 28 gives $G=0$ for $n=2$ while explaining the procedure in words to make clear that this method can be applied to the general case of arbitrary $n$ and $m$ :- Consider the top left elements of $G G^{*}$ and $G^{T} \bar{G}$ where $n=2$ :- all terms are positive and all elements of the top row of $G$ are present. The equations containing the top left and bottom right elements of $G \bar{G}$ may be combined as shown below to find that $G=0$.

For $n=2$ let

$$
G=\left(\begin{array}{cc}
z & y \\
x & w
\end{array}\right)
$$

the top left and bottom right equations are:

$$
\begin{array}{r}
3|z|^{2}+2|x|^{2}+y \bar{x}=0 \\
3|w|^{2}+2|y|^{2}+x \bar{y}=0
\end{array}
$$

and their combination is:

$$
3|z|^{2}+3|w|^{2}+|x|^{2}+|y|^{2}+|x+y|^{2}
$$

which means that $x=y=w=z=0$ in other words $G=0$. 
As mentioned, if $M=0$ then $G$ is a partial isometry. Equivalently, $G G^{*}$ and $G^{*} G$ are Murray-von-Neumann equivalent projections. And in the same way, we see for general $n, m$ the bottom $n-m$ rows of equation 28 show that the non-zero part of $G$ is a partial isometry.

Notice the simple relationship between $M$ and $G$; when $M^{*} M$ and $G^{*} G$ are diagonal projections and when $D_{F, v a c}^{2}=I$, they are the orthogonal complement of one another. The simultaneous matrix equations below simplify the statements: If $G=0$ then $M$ is a partial isometry and vice versa, and if $M=I$ then $G=0$ and vice versa, and if $M$ is a diagonal projection and if $D_{F, v a c}$ is invertible, then $G$ is a partial isometry orthogonal to $M$.

$$
\begin{aligned}
M\left(M^{*} M+G^{*} G-I\right) & =0 \\
G\left(G^{*} G+M^{*} M-I\right) & =0
\end{aligned}
$$

\subsection{Poincaré duality}

Given the standard model algebra and using Connes' realisation of Poincaré duality in Noncommutative geometry, we may write down the sign of the standard model Dirac operator. This is just the matrix [15] with eigenvalue ' 1 ' for each particle mass. An aim of the previous work was to answer the question, 'Is the standard model fermion mass matrix (internal space Dirac operator) a solution to the additional Einstein's equations?' whereas here we are considering the similar question, 'Is the sign of the standard model internal space Dirac operator a solution to the equations?' If the direction of this arrow is reversed, that is if the $K$-theory group is identified for a given vacuum solution, then topological information has been retrieved about the manifold from the minimisation procedure, and hence there would be a mathematical reason for the choice of the algebra up to Morita equivalence. We begin by exploring the relationship between vacuum solution and algebra. We only study the first two summands of the algebra, that is, the algebra for the Electroweak force. The matrix $M$ commutes with $\rho^{c}$ as the Higgs has no colour charge and we do not involve colour charge at all in the remainder of this article.

We study the standard model and one other solution.

Above a set of solutions was found where for a given projection $G$, the vacuum solution for $M$, or $M_{v a c}$ is determined via the simple relationship found in the equations of motion. Let us consider one such solution, namely the one in which $G=0$, that is, the one pertaining to the standard model. Then the $M_{v a c}^{*}$ is:

$$
M_{v a c}^{*}=\left(\begin{array}{ccc}
1 & 0 & 0 \\
0 & 1 & 0 \\
0 & 0 & 1 \\
0 & 0 & 0
\end{array}\right)
$$


which is a rectangular matrix because the two direct summands of the chirality $\mathbb{Z} / 2$-graded Hilbert space have different dimensions. The basis may be labelled $\left(u_{R}, d_{R}, e_{R}\right)^{T}$. We can add a final column of zeroes to $M_{v a c}^{*}$ and the basis becomes $\left(u_{R}, d_{R}, e_{R}, \nu_{R}\right)^{T}$.

Recall that $[F] \in \rho\left(\mathcal{A}^{o p}\right)$ where $\rho(\mathcal{A})$ is given by 3. The opposite algebra is represented by $J \rho(a) J^{-1}$. The generators of the $K$-homology group are the homotopy classes of the minimal rank projections of the opposite algebra $\mathcal{A}^{o}$, that is, $J \rho_{L}\left(p_{1}\right) J^{-1}$, which is the 2 by 2 unit matrix $\operatorname{diag}(1,1)$ and $J \rho_{R}\left(p_{2}\right) J^{-1}$, which is given by the number 1 . The former is a projection of the algebra of the quaternions $\mathbb{H}$ and the latter is simply a projection of the complex numbers $\mathbb{C}$. So the vacuum solution 29 is consistent with the element $b^{o}$ of the opposite algebra being:

$$
b^{o}=\left(\begin{array}{cccc}
\rho^{c T} & 0 & 0 & 0 \\
0 & \rho^{c T} & 0 & 0 \\
0 & 0 & q^{T} & 0 \\
0 & 0 & 0 & \Lambda^{T}
\end{array}\right)
$$

where $q$ is a quaternion and $\Lambda=\operatorname{diag}(\bar{\lambda}, \lambda), \lambda \in \mathbb{C}$. Using the reality operator we find that an element $b$ of $\mathcal{A}$ can be given by:

$$
b=\left(\begin{array}{cccc}
q & 0 & 0 & 0 \\
0 & \Lambda & 0 & 0 \\
0 & 0 & \rho^{c} & 0 \\
0 & 0 & 0 & \rho^{c}
\end{array}\right)
$$

from which we see that the first two summands of the algebra may be: $\mathbb{H} \oplus \mathbb{C}$.

Note that this is not a unique answer because the projection diag $(1,1)$ is also in $K_{0}(\mathbb{C})$.

A Fredholm module to be associated to a spectral triple must have algebra and Fredholm operator compatibility such that the first order condition is satisfied. In order to check that a spectral triple can be assigned to the Fredholm module, we check that the first order condition is satisfied. It is satisfied because $G=0$ and $\left[M, \rho^{c}\right]=0$ where $M$ is given by the direct sum.

We can generalise this procedure by choosing any other projective solution for $G$. For example let alternate eigenvalues of $G$ be non-zero beginning with the first eigenvalue zero. Then by the orthogonal complement relationship we have the solution for $M_{v a c}^{*}$ :

$$
M_{v a c}^{*}=\left(\begin{array}{ccc}
1 & 0 & 0 \\
0 & 0 & 0 \\
0 & 0 & 1 \\
0 & 0 & 0
\end{array}\right)
$$


and by the same procedure as above, we find that this solution is compatible with the algebra $M_{2}(\mathbb{C}) \oplus \mathbb{C} . M_{2}(\mathbb{C})$ is Morita equivalent to $\mathbb{C}$ and so the first two summands of the algebra of this spectral triple are Morita equivalent to $\mathbb{C} \oplus \mathbb{C}$.

A point of clarification is that the two solutions considered above are unitary (and homotopy $^{5}$ ) equivalent, and so one expects them lead to the same topological invariants. However, in the part to follow, we will restrict this homotopy freedom to separate 'nodes'; one for each of $\rho_{L}, \rho_{R}$ and $\rho^{c}$.

\subsection{Morse theory and the Witten complex}

The projection $\operatorname{diag}(1,1)$ is in the $K$-theory of both algebras $\mathbb{C}$ and $\mathbb{H}$, but to obtain topological information from the vacuum solutions the relationship between the vacuum solutions and the algebra must be one to one. We need a reason why the solutions should correspond only to the generators of the $K_{0}(\mathcal{A})$ group and not to any other element of the group. For example we need the matrix $\operatorname{diag}(1,1)$ to be associated only with $\mathbb{H}$ and algebras Morita equivalent to it.

In this section we generalise a theorem involving Morse theory and the Witten complex to suggest a method for finding topological information from the equations of motion about the $S^{o}$-real $(G=0)$ spectral triple pertaining to the standard model. The theorem is that a chain complex called the Witten complex, which is constructed from the critical points of the Morse function has the same homology groups as the manifold that the Morse function is defined on. Atiyah and Bott have proven that the Yang-Mills action is an equivariant Morse function and since the Higgs is a gauge field component in the internal space direction, we ask whether the finite spectral action is also a Morse function in a proposed noncommutative sense described below. To do this, we make a straightforward generalisation of the theorem to noncommutative geometry (which comes down to little more than the usual replacement of the commutative with the noncommutative algebra) by proposing a noncommutative generalisation to the definition of Morse function, equivariant Morse function (or Morse-Bott function), Hessian matrix and Witten complex.

A Morse function is a real-valued function on a (smooth) manifold $N, f: N \rightarrow$ $\mathbb{R}$ such that every one of its critical points is non-degenerate. The way to check for non-degeneracy is to calculate the Hessian matrix of the critical point and if this has no zero eigenvalues then the point is non-degenerate. Of course the Higgs vacuum is an entire three-sphere of non-degenerate solutions, so in order to use Morse theory in physics problems where there is a gauge symmetry, the equivariant Morse function was defined where the gauge symmetry is just divided out. (There are more complicated cases).

\footnotetext{
${ }^{5}$ unitary and homotopy equivalence are the same thing for stable algebras
} 
The Hessian matrix of a Morse function $f$ for a critical point is given by:

$$
a_{i j}=\left.\left(\frac{\partial^{2} f}{\partial x_{i} \partial x_{j}}\right)\right|_{c}
$$

where $c$ is the critical point, the $x_{i}$ are the coordinates on $N$ and $i$ runs from 1 to the dimension of $N$. The 'index' of the critical point is the number of negative eigenvalues of this matrix.

The action we want to consider is the finite spectral action with $G=0$ :

$$
S=\operatorname{Tr}\left(\left(M M^{*}\right)^{2}+-2 M M^{*}\right)
$$

To proceed from here, we need to make some generalisations of the above definitions to the context of noncommutative geometry. To begin with, the notion of a critical point is no longer valid, and differentiating with respect to each of the commuting coordinates $x_{i}$ on $N$ goes over to differentiating with respect to the matrix $M$. Consider the Fredholm operator $F$ ingredient of the Fredholm module over a noncommutative $C^{*}$-algebra. Recalling that $F$ is the generalisation to noncommutative geometry of an elliptic operator on a compact manifold in commutative geometry, we understand that $F$ is parametrised by the underlying space pertaining to the Fredholm module. Moreover, the homotopy classes of $F,[F] \in \rho\left(\mathcal{A}^{o p}\right)$. With in mind the Fredholm module picture of the underlying space on which the Morse function acts, we replace the $x_{i}$ with the $M$ as 2 by 2 matrices over $\mathbb{C}$. We use 2 by 2 matrices because each direct summand of the standard model algebra is viewed as a node (not quite a point) upon which the vector bundle is built, and the representation $\rho$ is separated into 2 by 2 'chunks'; one for each algebra summand. Obviously the critical points will be replaced by the vacuum solutions $M_{v a c}$. Finally we need to write down a condition corresponding to 33 that will give meaning to a noncommutative version of a Morse function, (or at least equivariant Morse function) and check that the action $S$ satisfies that condition. We continue this chapter's focus on the Fredholm module as the underlying space.

The index $i$ doesn't run very far because we are working in 0 dimensions and the action only depends on $M$ and its hermitian conjugate. The Hessian matrix can only be as follows:

$$
a=\left.\left(\frac{\partial^{2} S}{\partial M^{*} \partial M}\right)\right|_{c}
$$

$S$ is real-valued, and we don't need to worry about smoothness as this is already covered in the noncommutative geometry axioms. Differentiating the action $S$ with respect to $M$ and afterwards with respect to $M^{*}$ and evaluating at $M=0$ produces the Hessian of $S$. The resulting matrix is: $M M^{*}+M^{*} M-I$ evaluated at the vacuum solution which is that $M M^{*}$ and $M^{*} M$ are 'initial' and 'final' projections. 
Since in some solutions this matrix can have zero eigenvalues, we differentiate twice with respect to $M M^{*}$, so that $M M^{*}$ becomes the field to vary instead of $M$. The resulting matrix is $2 I$, which has no zero eigenvalues. We suggest then that $S$ is a noncommutative version of a Morse function. Since the degeneracies due to the vacuum manifold exist and may be associated with the finding of 0 eigenvalues above, it may be more accurate to designate the function as an equivariant Morse function in keeping with the analogy with Yang-Mills theory.

In (commutative) Morse theory, the Witten complex is defined as follows ([GT]). To begin, the free abelian group $C_{i}$ generated by the set of critical points of index $i$ is constructed. If $C_{i-1}$ is defined in the same way for index $i-1$, it is possible to define a map from $C_{i}$ to $C_{i-1}$, that is, the boundary map. This defines a chain complex called the Witten complex. It is a proven theorem that the homology groups of this chain complex are isomorphic to the homology groups of the manifold.

Classifying the vacuum solutions according to homotopy class, where each solution is homotopic to one of the following two diagonal projections:

$$
M_{v 1}=\left(\begin{array}{ll}
1 & 0 \\
0 & 1
\end{array}\right) \quad M_{v 2}=\left(\begin{array}{ll}
1 & 0 \\
0 & 0
\end{array}\right)
$$

this is equivalent to dividing out the degenerate solutions as in equivariant theory, and to considering the homotopy classes of the projections $(M M *)_{v}$ where the action is varied with respect to $M M^{*}$ instead of $M$.

Since the two solutions share the same Hessian, they have the same index and therefore the Witten complex for this particular function has only one component $C_{i}$ so the homology groups are simply $C_{i}$. Since the Witten chain complex in the case studied above is made out of only one term $C_{i}$, the kernel of the boundary map is $C_{i}$, and the image of the boundary map for the next term in the sequence takes the identity to the identity, therefore the homology group is simply given by $C_{i}$. The abelian group $C_{i}$ is generated by the two projections $M_{v 1}$ and $M_{v 2}$, which is exactly the $K$-homology group $K^{0}\left(\mathcal{A}^{o}\right)$, that is, the $K$-homology group of the underlying Fredholm module. (The other elements of the abelian group arise from the other generations - of which this framework implies there are an arbitrary number, and of course it is a mystery that we have to stop at 3 - in analogy to the abelian group $\mathbb{Z}$ where $S^{1}=\frac{\mathbb{R}}{\mathbb{Z}}$.) The $K$-theory group of the standard model algebra is isomorphic to $\mathbb{Z} \oplus \mathbb{Z} \oplus \mathbb{Z}$ (though here we have only studied the first two summands). Since one counts only a limited number of generations, the algebra is associated to the punctured torus.

The two 'critical nodes' can be identified with the first two direct summands (up to Morita equivalence) on the internal space, one for $\mathbb{H}$ and the other $\mathbb{C}$, an example of the noncommutative generalisation of the two point space, (left and right) as follows. We associate $M_{v 1}$ with left and $M_{v 2}$ with right. So $M_{v 1} \in \rho_{L}\left(\mathcal{A}^{o}\right)$ and $M_{v 2} \in \rho_{R}\left(\mathcal{A}^{o}\right)$. Removing $\nu_{R}$ from the Hilbert space and a row and column from $D_{F}$ means that the vacuum solution is: $M_{Q}=\operatorname{diag}(1,1)$ and $M_{L}=1$. These are the minimal rank 
projections of $\mathbb{H}$ and $\mathbb{C}$ and Morita equivalent algebras. And by Poincaré duality (as shown in the previous part), the noncommutative space can be described by the quaternions over the right 'node' and the complex numbers over the left.

\section{Conclusions}

Although the solutions to the field equations calculated in our previous work yielded an unphysical result, we have argued that the reasoning for their derivation deserves further attention and we explained why [SMV] has highlighted an open question in the Noncommutative framework. A solution was found to the equations of motion calculated previously with the 'leptoquarks' held constant whose features are compatible with the physical fermion mass matrix. Ironically, this result provides a mathematical reason for the Yukawa couplings to need fine-tuning.

The solutions to the field equations for $D_{F}$ both with and without the $S^{o}$-reality condition were shown to be partial isometries. These were interpreted as the phase or sign of $D_{F}$. The relationship between the vacuum solutions and the topology of the internal space was explored using $K$-theory and $K$-homology and a method inspired by Morse theory to extract topological information from the vacuum about the underlying Noncommutative space was developed. Instead of finding a mathematical reason in the action principle for the geometry of the standard model to be what it is known to be by experiment, we have claimed that the vacuum provides information on its topology in terms of $K$-homology. Rather than the vacuum picking out just one of a myriad of possible answers, all partial isometries are solutions. The study was limited to the first two of three summands, that is, the Electroweak part of the standard model algebra.

We also note that with $D_{F}^{2}=I$, we can consider solutions in the case without $S^{o_{-}}$ reality in which $M_{v 2}$ is associated with its orthogonal complement matrix $G_{v 2}=$ $\operatorname{diag}(0,1)$. Applying these solutions to the standard model basis, where $\nu_{R}=0$, $G=0$ and the bottom row vanishes from $M_{v 2}$ and the outcome is the same as above. Also, a similar analysis can be carried out with $M$ having arbitrary dimensionalities and arbitrary numbers of algebra direct summands. This involves either an unwanted prediction of new massless particles, or cutting the matrices down as above to fit the standard model Hilbert space. For example, in the dimensionality 3 case, there is an additional solution, $M_{v 3}=\operatorname{diag}(1,1,1) \in S U(3)$. Algebras with a greater number of summands cannot be identified using this method if there are repeated summands, for example the algebra $\mathbb{H} \oplus \mathbb{H} \oplus \mathbb{C} \oplus \mathbb{C}$ cannot be distinguished from $\mathbb{H} \oplus \mathbb{C}$ because they have the same critical 'nodes'.

\section{Acknowledgements}

Advices from J. W. Barrett and J. Zacharias were instrumental in the development of this research. The Engineering and Physical Sciences Research Council (EPSRC) 
are recognised for their financial support.

\section{Appendix}

The equations of motion for one generation and three coloured quarks with 'leptoquarks' held constant. These were calculated for [SMV] using the computer package Maple.

$$
\begin{gathered}
\bar{a}\left(3|a|^{2}+3|c|^{2}+3|b|^{2}+|g|^{2}+|u|^{2}+|x|^{2}+|h|^{2}+|v|^{2}+|y|^{2}-3\right)+3 \bar{c} \bar{b} d=0 \\
\bar{b}\left(3|b|^{2}+3|d|^{2}+|g|^{2}+|u|^{2}+|x|^{2}+|h|^{2}+|v|^{2}+|y|^{2}+3|a|^{2}-3\right)+3 \bar{a} \bar{d} c=0 \\
\bar{c}\left(|a|^{2}+|c|^{2}+|d|^{2}-1\right)+\bar{a} \bar{d} b=0 \\
\bar{d}\left(|b|^{2}+|d|^{2}+|c|^{2}-1\right)+\bar{c} \bar{b} a=0
\end{gathered}
$$

$\bar{q}\left(|q|^{2}+|s|^{2}+|r|^{2}+|g|^{2}+|u|^{2}+|x|^{2}+|j|^{2}-1\right)+\bar{r}(\bar{h} g+\bar{v} u+\bar{y} x+\bar{l} j+\bar{s} t)=0$

$$
\bar{r}\left(|r|^{2}+|t|^{2}+|q|^{2}+|h|^{2}+|v|^{2}+|y|^{2}+|l|^{2}-1\right)+\bar{q}(\bar{g} h+\bar{u} v+\bar{x} y+\bar{j} l+\bar{t} s)=0
$$

$$
\bar{s}\left(3|j|^{2}+|q|^{2}+|s|^{2}+|t|^{2}+|g|^{2}+|u|^{2}+|x|^{2}+|l|^{2}-1\right)+\bar{t}(\bar{h} g+\bar{v} u+\bar{y} x+2 \bar{l} j+\bar{q} r)=0
$$

$$
\bar{t}\left(3|l|^{2}+|r|^{2}+|t|^{2}+|s|^{2}+|h|^{2}+|v|^{2}+|y|^{2}+|j|^{2}-1\right)+\bar{s}(\bar{g} h+\bar{u} v+\bar{x} y+2 \bar{j} l+\bar{r} q)=0
$$

$\bar{j}\left(3|s|^{2}+|j|^{2}+|g|^{2}+|u|^{2}+|x|^{2}+|l|^{2}+|t|^{2}+|q|^{2}-1\right)+\bar{l}(\bar{r} q+2 \bar{t} s+\bar{g} h+\bar{u} v+\bar{x} y)=0$

$$
\bar{l}\left(3|t|^{2}+|h|^{2}+|v|^{2}+|y|^{2}+|j|^{2}+|l|^{2}+|r|^{2}+|s|^{2}-1\right)+\bar{j}(\bar{q} r+2 \bar{s} t+\bar{h} g+\bar{v} u+\bar{y} x)=0
$$




\section{References}

[AKH] N. Higson, J. Roe Analytic K-homology Oxford University Press (2000).

[BNG] A.W. Sitarz, The basics of Noncommutative geometry. url (visited June 2006) http://th-www.if.uj.edu.pl/ sitarz/pdf/habintro.pdf

[C] A. Connes Noncommutative Geometry. Academic Press, London, (1994).

[CFS] T. Krajewski, Classification of finite spectral triples. J. Geom. Phys. Vol.28 (1998), 1-30.

[FCG] T. Schücker, Forces from Connes' geometry. ArXiv:hep-th/0111236. Lect. Notes Phys. 659:285-350 (2005).

[GM] A. Connes, Gravity coupled with matter and the foundation of noncommutative geometry. Comm. Math. Phys. Vol.182 (1996), N.1, 155-176.

[IGT] M.A. Guest Morse theory in the 1990s., 'Invitations to Geometry and Topology' Edited by M.R. Bridson and S.M. Salamon. Oxford Science publications.

[INS] G. Landi, An introduction to noncommutative spaces and their topology. Arxiv: hep-th/9701078 (1997).

[L] G. Landi An Introduction to Noncommutative spaces and their geometries. Springer-Verlag (1998).

[NGL] M. Paschke, F. Scheck, A. Sitarz, Can (noncommutative) geometry accommodate leptoquarks? Arxiv: hep-th/9709009. Phys. Rev. D59:035003 (1999).

[NGR] A.Connes, Non-commutative geometry and reality. J. Math. Phys. Vol.36 (1995), 6194.

[NSM] T. Schücker, Noncommutative geometry and the standard model. Arxiv: hep-th/0409077. Int. J. Mod. Phys. A20:2471-2480 (2005).

[OCI] B. Iochum, T. Schücker, C. Stephan, On a classification of irreducible almost commutative geometries. J. Math. Phys. Vol.45 (2004), 5003-5041.

[SAP] A.H. Chamseddine, A. Connes, The spectral action principle. Comm. Math. Phys. Vol.186 (1997), N.3, 731-750.

[SMV] J.W. Barrett, R.A. Dawe Martins, Non-commutative geometry and the standard model vacuum. Arxiv: hep-th/0601192 (2006). To appear in J. Math. Phys.

[VBN] M. Khalkhali, Very basic noncommutative geometry. math-kt/0408416.

[W] N.E. Wegge-Olsen, $K$-theory and $C^{*}$-algebras. A friendly approach. Oxford Uni. Press (1993). 